Article

\title{
Assessing Outcomes of Online Training in Public Health: Changes in Individual and Organizational Knowledge and Capacity
}

John W. Wallace ${ }^{1}$, Jennifer A. Horney ${ }^{1, *}$, Rachel A. Wilfert ${ }^{2}$ and Lorraine K. Alexander ${ }^{1}$

1 Department of Epidemiology, Gillings School of Global Public Health, University of North Carolina at Chapel Hill, CB \# 7435, Chapel Hill, NC 27599, USA; E-Mails: jwwallac@email.unc.edu (J.W.W.); lorraine_alexander@unc.edu (L.K.A.)

2 North Carolina Institute for Public Health, Gillings School of Global Public Health, University of North Carolina at Chapel Hill, CB \# 8165, Chapel Hill, NC 27599, USA; E-Mail: rachel_wilfert@unc.edu

* Author to whom correspondence should be addressed; E-Mail: jen.horney@unc.edu; Tel.: +1-919-843-5566; Fax: +1-919-966-9138.

Received: 25 April 2013; in revised form: 4 September 2013 / Accepted: 23 September 2013 / Published: 27 September 2013

\begin{abstract}
The need for a well-prepared public health workforce to prepare for and respond to threats of terrorism, infectious diseases, and other public health emergencies is well documented, as is the reality that the public health workforce in the United States is under-trained and unprepared to handle public health emergencies. The impact of training on the public health workforce is often measured by the volume of training completed and post-course evaluation data. A survey of current, high-volume users $(n=759)$ of the University of North Carolina Center for Public Health Preparedness Training Web Site, defined as individuals who had completed 12 or more training modules was conducted in order to determine if measurable changes in preparedness and response knowledge and capacity were brought about by the trainings. Two-hundred and seventy respondents completed the survey (response rate $=36 \%$ ), with $52 \%$ reporting employment in governmental public health. Individual changes reported as a result of training included increased personal satisfaction (71\%), increased job satisfaction (38\%), and recognition by supervisors for training completion (23\%); Organizational changes included updates to training plans (19\%), making trainings mandatory (19\%), and revising standard operating procedures $(13 \%)$. Results from this survey indicate that the knowledge learned from completing online trainings led to changes in individuals and, to a lesser extent, changes in organizations.
\end{abstract}


Keywords: online; training; public health; preparedness; individual knowledge; organizational change

\section{Introduction}

The need for a well-prepared public health workforce to prepare for and respond to threats of terrorism, infectious diseases, and other public health emergencies is well documented, as is the reality that the public health workforce in the US is under-trained and unprepared to handle public health emergencies [1-4]. The University of North Carolina Center for Public Health Preparedness (UNC CPHP) was established as part of a national network of 27 Centers for Public Health Preparedness designed to improve the capacity of public health agencies and their staff through research, educational programs, and technical assistance. UNC CPHP has devoted much of its effort to creating Internet-based distance learning programs, which overcome many of the barriers to face-to-face training, including limited budgets, scheduling logistics, and travel restrictions that may prevent individuals from traveling to a physical training site [2,5]. UNC CPHP's long history with distance learning has also focused on addressing some of the limitations of online training, including ensuring effective instructional design, technology implementation, and evaluation.

The UNC CPHP Training Web Site (TWS) was created in 2003 to offer public health professionals and other interested individuals immediate access to short, 30- to 60-minute Internet-based training on public health preparedness topics including bioterrorism and disaster preparedness, public health surveillance, epidemiology, and emerging/reemerging infectious diseases. Trainings were developed by subject matter experts from the University of North Carolina at Chapel Hill Gilling's School of Global Public Health and peer-reviewed for content and appropriateness for distance learning. The TWS "justin-time" training is designed to aid the public health workforce in preparedness and response activities. The training content has evolved with the shifting focus of public health preparedness over the decade, beginning with bioterrorism, then hurricanes and natural disasters, and most recently pandemic influenza. As of August 2013, there were 258 trainings available for learners on the TWS; 83 additional trainings have been archived or made inactive as part of a continuous quality improvement review process. Throughout the span of the TWS there have been multiple reviews of training content, modifications to the TWS interface, and the inclusion of specific competency sets in training, currently including the Emergency Preparedness Competencies for all Public Health Workers developed by the Centers for Disease Control and Prevention (CDC) and the Columbia School of Nursing Center for Health Policy, Core Competencies for Public Health Professionals developed by the Public Health Foundation and the Council on Linkages, Public Health Preparedness and Response Core Competencies developed by the CDC and the Association of Schools of Public Health, and the CDC/CSTE Competencies for Applied Epidemiologists in Governmental Public Health Agencies developed by the CDC and the Council of State and Territorial Epidemiologists [6-9].

The impact of the TWS on the public health workforce is often measured by the volume of training completed and post-course evaluation data. As of August 9, 2010, over 88,000 training modules had been completed by 41,000 registered users from all 50 states and over 150 countries. The five most popular 
trainings on the TWS include training on the Health Insurance Portablity and Accountability Act, Cultural Competency, Introduction to Assessment, Bioterrorist Agents, and Introduction to Public Health Surveillance. Post-course evaluation data collected upon completion of each training module indicate very high user satisfaction with the content: $95 \%$ of users have stated that the training modules helped them feel better equipped to perform their professional roles and responsibilities and $91 \%$ felt the training modules addressed their professional roles and responsibilities. However, these data are indicators of satisfaction and knowledge improvement; they do little to inform us of actual changes in preparedness and response knowledge and capacity brought about by the UNC CPHP Training Web Site.

Since the goal of this site and of UNC CPHP is to improve the capacity of the public health workforce to respond to public health threats, we conducted a survey of high-volume users of the TWS to assess the effectiveness of the training modules in leading to individual and organizational change. By surveying users who had completed a substantial volume of training, we intended to capture individuals motivated to increase their knowledge and institute individual and organizational change. This approach was designed to assess, at least partially, Kirkpatrick's four conditions necessary for individual and organizational change: (1) the individual must be motivated to change; (2) the individual must know what and how to change; (3) the individual must be in a supportive climate for change; and, (4) there must be some reward, intrinsic or extrinsic, for changing [10].

\section{Experimental Section}

\subsection{Study Population}

In order to complete training modules offered on the TWS, users must first complete a registration form that collects their name, address, city, state, zip code, and email; then users are issued a login and a user-specified password. This login allows the user to access training content from a catalog of over 170 training modules on public health preparedness topics. Upon selecting a training module, users complete a pre-test quiz before accessing the training. The majority of training modules on the TWS are 30- or 60-minute modules consisting of synchronized audio and slide lecture in Adobe Presenter, formerly Macromedia Breeze format. Users must complete a post-test quiz made up of the same questions as the pre-test and a short end-of-course training evaluation, which assesses basic training-specific satisfaction and knowledge improvement. Participants also provide information on their occupation and the type of agency for which they work. Evaluation data are joined with registration data by user to create a training completion record which contains user demographic data, occupational data, and evaluation data for each training module completed. All data collected throughout the TWS are stored in a secured Oracle database housed in the Instructional and Informational Systems group at the UNC Gillings School of Global Public Health in Chapel Hill, NC. Only selected UNC CPHP staff have access to this database through a password-protected Internet-based administrative site which allows staff to query registrations, completions and evaluations, and other actions from the TWS.

The study population for the training impact survey consisted of current, high-volume TWS users, defined as individuals who had completed 12 or more training modules on the TWS before December 31,2010 . The cut point of 12 was determined based on the frequency distribution of the total number of training modules completed by individuals who visited the TWS (top quintile). High-volume users 
were selected to ensure that respondents would be familiar enough with the trainings to answer all questions completely. At least 1 training on or after January 1, 2008, was required to establish the user as a current, active training participant. Training completion data were queried from the Oracle database to obtain a total of 853 active, high-volume TWS users; they had completed a total of 20,825 training modules. While these individuals made up only 4\% of all TWS users from 2003-2010, their training completions comprised more than $25 \%$ of all training completed on the TWS.

\subsection{Survey Instrument}

In order to assess the use of the TWS by the public health workforce, the survey tool differentiated between academic users, both faculty and full-time students, who might access TWS materials for academic courses, and non-academic users, who are more likely to apply the knowledge and skills learned to their public health roles and responsibilities. Users reporting both academic and non-academic affiliations were classified as non-academic users.

All respondents were asked about access to the TWS, utility of training materials, their application of knowledge and skills learned through completion of training on the TWS, and the estimated number of individuals they had trained using TWS materials. Knowledge application was assessed by asking how respondents had applied the knowledge or materials from their training to their occupational settings.

Non-academic users were also asked questions about their satisfaction with and the perceived importance of TWS features that led to repeated use of the TWS. Additionally, these users were asked questions regarding individual and organizational changes that might have occurred based on TWS training completion, including new job responsibilities, promotions in the workplace, and motivation to find a new job or pursue certification or an advanced degree. The users were then asked to complete demographic information including occupation, type of agency in which they were employed, number of years worked in their current profession, highest degree earned, and state of residence. The survey instrument was developed in SurveyMonkey ${ }^{\mathrm{TM}}$ to allow Internet-based dissemination.

\subsection{Survey Method}

In March 2010 a pilot survey was sent out via email to 54 randomly selected individuals in the study population. In an attempt to increase the notoriously poor response rates of Internet-based surveys, the email included information on how to register for a drawing for a small incentive. A follow-up reminder email was sent a week after the initial email, and the survey was closed after 10 days. Four emails could not be delivered, and of the 50 successfully delivered emails, 13 pilot surveys were completed, for a response-rate of $26 \%$. Results from the pilot survey were used to better tailor the survey instrument to the study population (e.g., survey was edited for clarity; no major changes were made) and were not included in the analysis of survey data.

The final survey instrument was disseminated via email to the remaining 799 individuals in the study population; again, the email included information on how to register for a drawing for a small incentive. The survey remained open for 3 full weeks, with 2 reminder emails sent every 7 days. Upon closure of the survey, a drawing was held for the incentive and the data were downloaded from SurveyMonkey ${ }^{\mathrm{TM}}$, then cleaned and analyzed using SAS 9.2 (Cary, NC). Data analysis consisted primarily of frequency distributions for each survey question, with academic and non-academic respondents 
analyzed separately. Demographics were compared between survey respondents and current TWS users using Pearson's Chi-Square test. The potential reach of training materials was calculated by asking respondents to report the number of people with whom they shared knowledge or materials from the TWS $(0,1-5,6-20$, and 20 or more), and using the minimum value of the range selected to calculate a total reach.3. Results and Discussion

Of the 799 individuals who were sent the survey, 759 received the initial email and the two reminder emails. The remaining 40 individuals were determined to have undeliverable email addresses due to a returned email. Two hundred seventy of the 759 individuals responded to the survey, a $36 \%$ response rate. Two hundred forty-four (90\%) individuals were classified as non-academic users, reporting they were either part-time students at a college or university $(8.9 \%)$ or their primary affiliation was not with a college or university $(82 \%)$, and the remaining 26 respondents $(10 \%)$ were classified as academic users.

\subsection{Academic Users}

Knowledge Application

Knowledge application of academic users was assessed by asking respondents how they had applied the knowledge or materials from TWS training modules to their academic work. Twenty-five of the 26 academic users provided responses to knowledge application questions. Eighty-four percent of the 25 academic users reported that they had applied the skills learned from the Training Web Site to their studies or teaching, and $96 \%$ had shared information. When asked specifically how the knowledge learned from the training modules had been used, respondents indicated use in academic courses (64\%), practicums $(24 \%)$, and seminars $(20 \%)$. Users were also asked to estimate the number of individuals with whom they had shared knowledge or materials from the TWS. Of the 24 academic respondents, half $(\mathrm{n}=12)$ reported sharing knowledge with $1-5$ individuals, $17 \%(\mathrm{n}=4)$ with $6-20$, and a third $(\mathrm{n}=8)$ reported sharing knowledge with more than 20 individuals. These 24 respondents shared knowledge with a minimum of 204 individuals, an average of $8.5(\mathrm{SD}=9.0)$ additional individuals reached for each high volume academic user.

\subsection{Non-Academic Users}

\subsubsection{Demographics}

Table 1 compares the demographic characteristics of the 244 non-academic survey respondents to the total population of high-volume current TWS users and the general population of TWS users. Over one-quarter were from North Carolina; $12.7 \%$ were from 20 countries outside the United States, in North America, South America, Europe, Africa, and Asia.

Nurses made up the majority of survey respondents, with $22.1 \%$ self-reporting their occupation in the field of nursing. Over half the respondents were employed in governmental public health at the state, local, or federal level, and almost $65 \%$ had earned a bachelor's degree or higher. The survey respondents were more highly educated $\left(\chi^{2}=52.8 \mathrm{df}=1, p<0.0001\right)$ and more likely to work in governmental public health $\left(\chi^{2}=5.76, \mathrm{df}=1, p=0.02\right)$ than other current TWS users. 
Table 1. Demographic characteristics of survey respondents compared to all high-volume current Training Web Site (TWS) users and overall current TWS users.

\begin{tabular}{lccc}
\hline & $\begin{array}{c}\text { Survey Respondents } \\
(\mathbf{\%}) \mathbf{( n = 2 4 4 )}\end{array}$ & $\begin{array}{c}\text { High-volume Current } \\
\text { Users } \mathbf{( \% ) ( n = 8 9 8 )}\end{array}$ & $\begin{array}{c}\text { Current Users } \\
(\mathbf{\%}) \mathbf{( n = 9 , 3 0 9 )}\end{array}$ \\
\hline North Carolina Users & 26.6 & 21.4 & 24.3 \\
\hline Other US Users & 55.4 & 68.8 & 67.0 \\
\hline International Users & 12.7 & 9.8 & 26.5 \\
\hline $\begin{array}{l}\text { Nurse Practitioner/Licensed } \\
\text { Practical Nurse/Registered } \\
\text { Nurse }\end{array}$ & 22.1 & 21.0 & \\
\hline $\begin{array}{l}\text { Government Public Health } \\
\text { Employee (Local, State, }\end{array}$ & 52.1 & & 44.3 \\
Federal) & & 46.9 & 41.5 \\
\hline Bachelor's degree or higher & 64.8 & 45.4 & 12.9 \\
\hline Associate Degree & 20.1 & 10.9 & 24.0 \\
\hline Bachelor's Degree & 30.7 & 22.5 & 13.3 \\
\hline Master's Degree & 26.2 & 16.8 & 4.2 \\
\hline Doctoral Degree & 7.8 & 6.1 & \\
\hline
\end{tabular}

\subsubsection{Satisfaction with the Training Web Site}

Users were asked about the TWS features that led to return visits to the site and their satisfaction with these features. Useful, up-to-date information (100\%), no cost training modules (99\%), and training on specific public health preparedness topics (94.1\%) were among the most important reasons individuals returned to the TWS. Additionally, 97\% of respondents indicated that the ease of finding appropriate training was either important or very important. The features that were least important for returning to the site were the opportunity to earn continuing education units $(24.5 \%)$ and the ability to revisit previously completed training $(22.4 \%)$.

Respondents were most satisfied with the provision of no cost training modules $(99.6 \%)$, the length of training modules (95.7\%), and the useful, up-to-date information (95.3\%). They were least satisfied with the ease of finding appropriate training modules $(12.8 \%)$, the opportunity to earn continuing education units $(7.0 \%)$, and the provision of training on specific public health preparedness topics (6.8\%), even though both ease of finding and provision of training on a specific public health preparedness topic were both listed as one of the more important features of the TWS related to return visits. 


\subsubsection{Knowledge Application}

Knowledge application was assessed based on how respondents had applied the knowledge or materials from TWS training modules to their occupational setting. Seventy-five percent of nonacademic respondents had applied skills and knowledge learned from the TWS to their job or career, and $78 \%$ had shared knowledge or material from TWS training modules with coworkers (Table 2). When directly compared to academic users, non-academic users were less likely to share information ( $78 \%$ vs $\left.96 \% ; \chi^{2}=4.40, \mathrm{df}=1, p=0.04\right)$ but no more likely to apply knowledge or material from the TWS $\left(75 \%\right.$ vs $\left.84 \% ; \chi^{2}=1.08, \mathrm{df}=1, p=0.30\right)$. When asked with whom knowledge had been shared, respondents said they had shared in a group setting (58\%), in one-on-one assistance or support (50\%), through material distribution (28\%), or through formal trainings $(15 \%)$. When non-academic users were asked to estimate the number of individuals with whom knowledge or materials from the TWS had been shared, over half $(52 \%, \mathrm{n}=102)$ said they had shared knowledge with $1-5$ individuals, $31 \%$ $(\mathrm{n}=61)$ with 6-20 individuals, and $17 \%(\mathrm{n}=33)$ with more than 20 individuals. Thus these 196 respondents potentially had shared knowledge with at least 1,181 individuals, corresponding to 6 additional individuals reached per one high-volume user $(\mathrm{SD}=7.2)$.

Table 2. Application and Sharing of TWS Knowledge by Non-Academic Users ( $\mathrm{n}=244)$.

\begin{tabular}{cccc}
\hline & Frequency & Percent & \\
\cline { 1 - 3 } Applied TWS skills to job or career & 173 & $75 \%$ & \\
Shared information & 181 & $78 \%$ & \\
\hline & & & \\
\hline Number of individuals with whom & Frequency & Percent & $\begin{array}{c}\text { Minimum number of } \\
\text { individuals reached }\end{array}$ \\
knowledge was shared & 101 & $52 \%$ & 101 \\
$1-5$ individuals & 61 & $31 \%$ & 366 \\
6-20 individuals & 34 & $17 \%$ & 714 \\
\hline 20 individuals & & &
\end{tabular}

\subsubsection{Individual/Organizational Change}

Forty-six percent of non-academic respondents (108) reported that the training modules had helped them perform new job responsibilities, and 31\% said the training had motivated them to pursue certifications or an advanced degree in public health. When presented with a list of individual changes that might have resulted from the training, a substantial number of respondents (71\%) reported increased personal satisfaction, and 38\% reported increased satisfaction with their current job. Twenty-three percent noted recognition for accomplishments, and $18 \%$ reported an increase in job responsibilities. However, $18 \%$ said, "None of the above," implying that no individual changes had occurred (Table 3).

Non-academic respondents were also asked if training on the NCCPHP web site was required or recommended, and 19\% reported that training was required, 30\% responded that it was recommended but not required, and the remaining 51\% indicated that completing TWS training was neither required nor recommended. In assessing organizational change, 19\% reported that their organization had begun requiring training for certain staff in the organization as a result of the training the user had completed on the TWS. Similarly, 19\% reported that new or additional training had been added to their organization's 
training plan as a result of TWS training completed. Thirteen percent reported changes in standard operating procedures and 5.3\% reported changes in policies based on knowledge gained from training modules. However, 65\% (147 respondents) reported no organizational changes.

Table 3. Individual and Organizational Changes among non-academic survey respondents.

\begin{tabular}{lcc}
\hline Individual Changes & Frequency & Percent \\
\hline Improved personal satisfaction & 165 & $71 \%$ \\
Improved satisfaction with current job & 89 & $38 \%$ \\
Recognition for accomplishments & 53 & $23 \%$ \\
Increase or upgrade in job responsibilities & 42 & $18 \%$ \\
Desire to find a new job & 29 & $12 \%$ \\
Promotion or new job & 15 & $6.4 \%$ \\
Increase in salary & 12 & $5.2 \%$ \\
Other* & 7 & $3.0 \%$ \\
None of the above* & 41 & $18 \%$ \\
\hline Organizational Changes & & \\
\hline Included new/additional training in training plan & 44 & $19 \%$ \\
Required training within the organization for certain staff & 44 & $19 \%$ \\
Revised standard operating procedures based on knowledge learned & 29 & $13 \%$ \\
from the training completed & & \\
Changed policies based on knowledge learned from the training & 12 & $5.3 \%$ \\
completed & & $2.2 \%$ \\
Other* & 5 & $65 \%$ \\
None of the above* & 147 & \\
\hline
\end{tabular}

* The "Other" response indicates a change did occur but not one presented in this list, while the "None of the above" response indicates no change.

\section{Conclusions}

This TWS high-volume user survey explored the use of knowledge and materials gained from TWS training. While the response rate was relatively low, it was typical for online surveys, which have been shown in reviews to have a mean response rate of $36.83 \%$ [11]. We obtained valuable information on how the UNC CPHP Training Web Site increases user knowledge and leads to individual changes and ultimately to sharing of knowledge with other individuals and some organizational changes.

\subsection{Satisfaction with the Training Web Site}

User satisfaction with the TWS is always reported to be very high in end-of-course evaluations, and this was confirmed by our survey of high-volume users. The user satisfaction section in the survey assessed the features of the TWS that users believed to be important drivers of return visits and their satisfaction with these features. It was not surprising to find that training modules provided at no cost was one of the most important and most satisfactory features of the TWS, along with the provision of useful, up-to-date information. Another important feature, however, the ease of finding appropriate training, was considered one of the least satisfactory features of the TWS. The UNC CPHP has begun to address this issue by creating searches by MESH keyword, public health topic, and public health 
competency set, but the stated need for improvement points to the need for a more efficient cataloging and search system.

The feature that the highest proportion of respondents considered not important or only somewhat important was the opportunity to earn continuing education units. UNC CPHP has provided free Continuing Education Units (CEUs) to users who wish to receive credit for training, but we have heard anecdotally from users that more occupation-specific CEUs, such as Continuing Medical Education credits and Environmental Health Specialist credits, are more desirable. Based on this feedback, UNC CPHP has worked to provide credits specifically for nurses, Certified Health Education Specialists, and registered sanitarians / environmental health scientists. The ability to revisit previously completed trainings was also considered one of the least important features, though we think it is necessary to provide users the ability to return to the training for reference at a later time. High technical quality of presentations was also less important than most features, with $14.5 \%$ indicating either not important or somewhat important. This suggests that users visit the TWS for the material, and the method of delivery is secondary. Nevertheless, as part of our continuing quality improvement effort, trainings are frequently reviewed and archived if they are deemed to be no longer scientifically accurate or of high technical quality.

\subsection{Knowledge Application}

Both academic and non-academic respondents were presented with questions related to their application of knowledge and materials from the TWS, and a large percentage of both types of users applied the knowledge learned from the TWS to their occupation. However, proportionately more academic users shared information. This difference could be attributable to the use of TWS in academic courses, which is consistent with anecdotal evidence obtained previously by the authors through informal requests from instructors to use trainings as part of academic courses. This may also reflect the growing number of public health programs, both for continuing education and for academic credit, that are now offered in an online format.

The use of TWS knowledge in academic courses may also explain the difference in the number of individuals with whom TWS knowledge was shared. Nevertheless, both academic and non-academic survey respondents were able to share the knowledge they had learned with a large number of individuals, increasing the reach of the TWS to the public health workforce. This is another way in which the training modules may be cost-effective during these times of fiscal constraint, by facilitating sharing of information without requiring additional staff to participate in the training in its entirety.

\subsection{Individual/Organizational Change}

Almost half of the non-academic survey respondents indicated that the knowledge gained from the TWS helped them perform new job responsibilities. Increased personal satisfaction and job satisfaction were the most commonly reported individual changes. Not unexpectedly, very few individuals reported an increase in salary or a promotion as a result of their training, though some respondents did report increased or upgraded job responsibilities and a desire to find a new job, and motivation to pursue certifications or an advanced degree in the field of public health. These findings suggest that training 
sites such as the TWS may bring about intrinsic changes not measurable by performance indicators such as promotions or salary increases.

Fewer individuals reported that the training completed on the TWS resulted in organizational change, although some did indicate that their organization began requiring training for certain staff in the organization or added new topics to their organization's training plan. While the organizational changes were relatively small, it can be concluded that the TWS training does affect the training environment in the workplace. This could lead to further training and increases in public health capacities.

\subsection{Limitations}

There were several limitations to our approach. First, the data were all self-reported, as is the nature of most Internet-based research. However, there was little reason for respondents to withhold information or not accurately respond to the questions. The survey instrument did not contain any questions that were of a sensitive nature, and although training behaviors were assessed, no identifying data were collected that could link respondents to their surveys. Further, there was little or no motivation for respondents to embellish their use of the TWS or the application of their knowledge.

Second, we purposely selected a group of very motivated users who had completed 12 or more training modules on the TWS, in order to assess the use of the training. As expected, the responses were generally favorable towards use and application of knowledge learned through the TWS, but these responses may not be representative of the entire population of current TWS users. Also as Table 1 shows, the survey respondents were more educated and more likely to work in governmental public health than either the target population of high-volume, current TWS users or the broader group of current TWS users. Inevitably, these differences and the study design itself led to a selection bias towards individuals who are more likely to respond to Internet-based surveys and more likely to share knowledge. In addition, we may have overestimated the additional reach of TWS materials. We cannot ensure that the individuals that users reported sharing knowledge with were unique.

Finally, our method of evaluating changes at individual and organizational levels through a survey of trainees is not ideal for assessing such changes. Kirkpatrick identifies several methods of measuring change in evaluating training programs, one of which is to interview not only the trainees, but also their supervisor(s) and/or subordinate(s) who might notice these changes. This approach would provide a more objective assessment of changes that resulted from training completed on the TWS [10].

Despite these limitations, the results from this survey indicate that the knowledge learned from completing TWS training led to changes in individuals and, to a lesser extent, changes in organizations. The TWS provides an opportunity for motivated individuals, like our high-volume user survey respondents, to find training to increase their public health knowledge. Further, many of these individuals reported some sort of reward, including improved personal satisfaction or recognition for their achievements, which indicates a favorable climate for change. Clearly this training site and other similar training sites have created a favorable work environment where training is seen as an opportunity to increase knowledge of public health topics. Thus, while the short, 30- to 60-minute training modules on the TWS may not lead directly to large-scale organizational changes, we believe the UNC CPHP Training Web Site serves as an important tool for increasing the capacity of the public health workforce to respond to public health threats. 


\section{Acknowledgement}

This publication was supported by Grant/Cooperative Agreement Number U90TP000415from the Centers for Disease Control and Prevention (CDC). Its contents are solely the responsibility of the authors and do not necessarily represent the official views of CDC.

\section{Conflicts of Interest}

The authors declare no conflict of interest.

\section{References}

1. Trust for America's health. In Ready or Not? Protecting the Public's Health in the Age of Bioterrorism; Robert Wood Johnson Foundation: Princeton, NJ, USA, 2012; Available online: http:/healthyamericans.org/assets/files/TFAH2012ReadyorNot10.pdf (accessed on 20 August 2013).

2. Public health workforce study. In National Center for Health Workforce Analysis; Health Resources and Services Administration: Rockville, MD, USA, 2005; Available online: http://bhpr.hrsa.gov/ healthworkforce/reports/publichealthstudy2005.pdf (accessed on 20 August 2013).

3. Botoseneanu, A.; Wu, H.; Wasserman, J.; Jacobson, P.D. Achieving public health legal preparedness: How dissonant views on public health law threaten emergency preparedness and response. $J$. Public Health 2011, 33, 361-368.

4. Nelson, C.; Laurie , N.; Wasserman, J.; Zakowski, S. Conceptualizing and defining public health preparedness. Am. J. Public Health 2007, 97, S9-S11.

5. Steckler, A.; Farel, A.; Bontempi, J.B.; Umble, K.; Polhamus, B.; Trester, A. Can health professionals learn qualitative evaluation methods on the World Wide Web? A case example. Health Educ. Res. 2001, 16, 735-745.

6. Bioterrorism and Emergency Readiness: Competencies for All Public Health Workers; Columbia University School of Nursing Center for Health Policy: New York, NY, USA, 2002; Available online:http://raining.fema.gov/emiweb/downloads/BioTerrorism\%20and\%20Emergency\%20Rea diness.pdf (accessed on 2 February 2012).

7. Core Competencies for Public Health Professionals; Council on Linkages Between Academia and Public Health Practice: Washington, Washington DC, USA, 2010; Available online: http://www.phf.org/programs/corecompetencies/Pages/About the_Core_Competencies_for_Public_ Health_Professionals.aspx (accessed on 2 February 2012).

8. Public Health Preparedness and Response Core Competency Model; Centers for Disease Control and Prevention and Association of Schools of Public Health: Washington, Washington DC, USA, 2011; Available online: http://www.asph.org/document.cfm?page=1083\#Preparedness/ (accessed on 2 February 2012).

9. Competencies for Applied Epidemiologists in Governmental Public Health Agencies (AECs); Centers for Disease Control and Prevention and Council of State and Territorial Epidemiologists: Washington, Washington DC, USA, 2008; Available online: http://www.cdc.gov/ AppliedEpiCompetencies/downloads/Applied_Epi_Comps.pdf (accessed on 2 February 2012). 
10. Evaluating Training Programs: The Four Levels, Kirkpatrick, D.L., Ed.; Berrett-Koehler Publishers: San Francisco, CA, USA, 1998.

11. Sheehan, K.B. Email survey response rates: A review. J. Comput.-Mediat. Commun. 2006, 6, doi:10.1111/j.1083-6101.2001.tb00117.x.

(C) 2013 by the authors; licensee MDPI, Basel, Switzerland. This article is an open access article distributed under the terms and conditions of the Creative Commons Attribution license (http://creativecommons.org/licenses/by/3.0/). 